\title{
Effects of gender inequality and income inequality on within-sex mating competition under hypergyny
}

\author{
Robert C. Brooks ${ }^{1}$, Khandis R. Blake ${ }^{1,2}$ \& Lutz Fromhage ${ }^{3}$
}

1. Evolution \& Ecology Research Centre, UNSW Sydney, 2052, N.S.W. Australia

2. Melbourne School of Psychological Sciences, The University of Melbourne

3. Department of Biological and Environmental Science, University of Jyvaskyla, Finland

\begin{abstract}
Author Note
rob.brooks@unsw.edu.au, khandis.blake@unimelb.edu.au, lutz.fromhage@jyu.fi
\end{abstract}

\section{Acknowledgments}

We acknowledge the support of the Australian Research Council (Discovery Grant DP190100899). This work was greatly improved by discussions with Michael Jennions and Francesca Luberti and by the comments of two anonymous referees. 


\title{
Effects of gender inequality and income inequality on within-sex mating competition under hypergyny
}

\begin{abstract}
Resources are often central to the formation and persistence of human consortships, and to the evolutionary fitness consequences of those consortships. As a result, the distribution of resources within a society should influence the number and quality of mating opportunities an individual of given status/wealth experiences. In particular, in a wide variety of societies, both contemporary and historic, women have been shown to prefer mates of higher rather than lower status and wealth, a pattern known as 'hypergyny'. Such status-dependent within-sex competition is likely to be influenced not only by the preferences individuals express but also by the distribution of resources within and between sexes. Empirical studies show that economic inequality within a sex can amplify mating competition, and that inequalities between women and men also influence behaviours related to mating competition, but the links between resource distribution and mating competition have attracted limited systematic attention. We present simulation models of hypergynous preferences and the effects on mating competition among men and among women within a heterosexual mating market. Our modelling shows that the lower mating success of poorer men and richer women (when compared with richer men and poorer women) is worsened when resource gender gaps are relatively small or when women out-earn men on average. Likewise, high economic inequality, especially among men, amplifies the competition experienced by these groups. We consider the political implications in terms of sex-and status-dependent attitudes to gender equity, income inequality, and hypergynous mating norms.
\end{abstract}




\section{Introduction}

Economic considerations and incentives have shaped the evolution of human mate choice. A consortship is, inter alia, an economic arrangement, in which both parties share, to some degree at least, their wealth, income, time, and labour (Becker 1973, 1981, GrossbardShechtman 1984, Chiappori et al. 2002). Human mating preferences and strategies have evolved a fine tuning to this economic dimension of mating, biasing individuals to choose mates who have, or are likely to have, the attributes likely to lead to a successful economic partnership (Buss 1991, Buss et al. 2001, Hopcroft 2006, Hopcroft 2021).

Data from traditional, historic, and contemporary societies show that male status and wealth are often strongly associated with success in marriage, extra-marital mating, and producing offspring (Voland 1990, Cronk 1991, see review by Hopcroft 2006, Weeden et al. 2006, Nettle and Pollet 2008, von Rueden and Jaeggi 2016, Hopcroft 2021). The effects of status on female mating success are more variegated; women of higher status or greater wealth often bear fewer children (Hopcroft 2006). Those children, however, are more likely to survive early childhood (Boone III 1986, Hughes 1986, Lummaa 2001, Lartey et al. 2016), suggesting that for women, too, status elevates evolutionary fitness.

Part of the fitness benefit that high-status men enjoy derives from the practice of hypergyny, according to which women are more likely to "marry upward" to men of higher status than themselves, or to sons of families of higher status than their own. Even though individual women can - and occasionally do - pair with men of lower status, it is far more common for women to pair with men of equal or higher status. As a result, hypergyny is a widespread pattern across contemporary and historic societies (Dickemann 1979, Betzig 1986, Boone III 1986, Betzig 1994, Wooding et al. 2004, Nettle and Pollet 2008, Van Leeuwen and Maas 2010), whereas the reverse pattern (hypogyny - women pairing downward) is unheard of at an aggregate level.

Adaptive theories of human mating emphasise Trivers' (1972) argument that sex differences in obligate parental investment drive the evolution of mate choice and within-sex competition. In humans, 'parental investment theory' is often invoked to explain women's 
preference for hypergyny and thus men's competition for wealth and status (Buss and Schmitt 1993, Buss 1995). Trivers' view has, in the intervening half century, been developed into less static models (Kokko and Jennions 2008, Fromhage and Jennions 2016) that better account for among-and within-species variation in animal sex roles and mating behaviour. The promise of this more dynamic approach has, to date, yet to be realised in human research on questions like how cultural variation in the strength of hypergyny arises and how it is enabled or enforced culturally.

The primary aim of this paper is to predict how economic inequalities shape within-sex competition. Considerable evidence points to the importance of economic inequality within a sex on competitive behaviour (Wilson and Daly 1997, Daly and Wilson 2001, Daly 2016, Blake et al. 2018, Blake and Brooks 2019). Likewise inequalities between women and men have been shown to influence behaviours related to mating competition (Price et al. 2014). Despite the importance of inequalities to human mating markets, most thinking on the subject comprises verbal models and interpretation of empirical evidence. Here we present a simple simulation model of hypergynous preference and describe how hypergyny predicts competition among men to mate with women, and among women to mate with men. It predicts how these forms of competition are likely to be affected by economic inequalities among men, among women, and between women and men. Our aim is to lay a foundation for a more formal and integrated adaptationist study of how inequalities shape sexual competition and its various manifestations, some of which have important psychological, safety, public health, and security implications.

\section{Lessons from the study of sex ratios}

In contrast to the limited modelling of economic inequality's effects on mating competition, the effects of sex ratios on mating competition have received extensive theoretic development and review by biologists (Emlen and Oring 1977, Shuster and Wade 2003, Kokko and Jennions 2008, Klug et al. 2010, de Jong et al. 2012, Kokko et al. 2012, Shuster 2016). Operational Sex Ratios (OSR) - defined by Emlen (1976) as "the ratio of receptive females to potential mating males at any one time" - are widely used in studies of animal mating systems and sexual selection (Kvarnemo and Ahnesjo 1996), spawning, according to Shuster (2016), over 4500 articles. In parallel, the effects of relative numbers of women and 
men (i.e., human adult sex ratio) on within-sex mating competition have been subjects of theoretic modelling and empirical research in the human behavioural sciences (GrossbardShechtman 1984, Chiappori et al. 2002, Kandrik et al. 2015)(Guttentag and Secord 1983, Marlowe and Berbesque 2012, Schacht and Borgerhoff Mulder 2015, Stone 2018).

Sex ratios affect mating markets by altering the supply of mates relative to competitors. Under male-biased sex ratios, women enjoy greater bargaining power over the establishment of relationships, as well as within relationships (Guttentag and Secord 1983), leading to higher rates of marriage, lower rates of births to teen and unwed mothers, and better mate choice outcomes for women (Barber 2000, reviewed by Stone 2018).

Male-biased sex ratios intensify competition among men, and some sources show this elevates rates of violence, property crime, addiction, and radicalisation (Hudson and Boer 2004, Edlund et al. 2010). Other studies show that when sex ratios are female-biased, men invest less in their consorts and children, invest more in mating competition (Stone 2018), are more open to uncommitted sex (Kandrik et al. 2015) and to 'poaching' mates from other men (Schmitt and Descript 2004). These forms of elevated mating competition stoke maleperpetrated violence in some societies when men are relatively rare (Schacht et al. 2016). It is clear from the ample work on sex ratios that the effects are manifold, if somewhat complex.

\section{Context-dependent competition}

The effects of sex ratios, or any other variable, on within-sex competition are seldom uniform in their expression. In general, young unmarried men who are poor, unemployed, and thus have dim prospects of finding a partner, experience the most intense competition. As a result of this competition, violent offending, dangerous driving, gambling and other risky behaviours are highest among these men (Wilson and Daly 1985, Daly and Wilson 2001). Wilson and Daly (1985) named this constellation of traits "young male syndrome", arguing that these traits function adaptively to enhance status as a means to outcompeting sexual rivals. 
Factors that perturb mating markets have a disproportionate effect on young, poor, uneducated men, especially those from low-status groups. A male-biased sex ratio exacerbates the intense competition among low-status men who massively outnumber available women, whereas the mating prospects of high-status men remain largely unaffected. As a result, it is often the poorest men, ceteris paribus, who are at greatest risk of being unable to mate, and thus of falling into violence, crime, addiction, and chronic physical and mental illness (Hudson 2002, Hesketh and Xing 2006, Zhou et al. 2013).

Competition among women is also not uniform. Women in low socioeconomic status (SES) neighbourhoods in England reproduce at younger ages when sex ratios are female-biased, whereas women from high SES neighbourhoods do so at older ages (Chipman and Morrison 2013). The interpretation is that, when mates are rare, poorer women cannot afford to wait for a high-earning mate, whereas richer women can do so, and often do. Likewise, when women outnumber men, it is often high-earning or high-status women who are at greatest risk of remaining unpartnered.

\section{Varying effects of income inequality}

Just as the supply-demand effects of sex ratios exert uneven effects on competition, the distribution of wealth can have comparable effects. The most studied such effect is that of income inequality on male-male competition. Income inequality predicts risky male behaviours, violent crime, and homicide (Krahn et al. 1986, Wilson and Daly 1997, Daly et al. 2001, Peñaherrera-Aguirre et al. 2018). Martin Daly and Margo Wilson marshalled a number of strands of evidence showing that these links are due to an intensification of male-male competition under high economic inequality, particularly for poorer, younger men (Wilson and Daly 1997, Daly et al. 2001, Daly 2016, see also Greitemeyer and Sagioglou 2017).

Competition among women, too, responds to economic inequality. Teenage and unwed births are associated with high inequality (Wilson and Daly 1997). Recently we showed that women's self-sexualisation in the form of posting 'sexy selfies' on social media is robustly associated with high economic inequality at the level of U.S. cities, U.S. counties, and nations (Blake et al. 2018). Moreover, at the U.S. city and county level, women's expenditure on clothing was associated positively and robustly with inequality (Blake et al. 
2018). Whether the effects of inequality on self-sexualisation, grooming, and other expressions of women's competition are uniform, or if they are particularly strong in some women rather than others, currently remains unresolved (Blake and Brooks 2019). Part of the rationale for this study is to model, under simplified conditions, the mating success implications of economic inequalities within and between the sexes for women and men in relation to their status/wealth.

Income or wealth inequality is most often measured and studied at the household level, with little heed paid to the possibility that inequalities among men and among women might exert different effects on behaviour. Within $20^{\text {th }}$ Century Western economies, inequalities changed from the 1920s (high gender inequality and income inequality among men, low income inequality among women) to the 1950s (high gender inequality, low income inequality within each sex) to the 1980s (lower gender inequality, high inequality within both sexes), with effects on mating markets and gender relations that remain contested (Goldin 1990, Stevenson and Wolfers 2007). Theory concerning whether and how the effects of inequality among men and among women might differ remains limited, at best. One of our aims, here, is to begin the process of dissecting the likely effects of economic inequality on each sex.

\section{Gender inequality}

Inequality between the sexes has profound and pervasive effects on human behaviour, including on mating systems and norms concerning sex and the family (Reiss 1986, Jetten and Peters 2019). Differences between women and men in wealth, earning potential, status, and power define the landscape of relations between the sexes (Stevenson and Wolfers 2007, Bertrand et al. 2013, Price et al. 2014, Autor et al. 2019). Nonetheless, few published accounts explicitly consider how gender inequality influences the intensity of competition within each sex.

The more men's mean incomes exceed those earned by women, the greater the return from marriage that women experience (Becker 1973, Wilson 1996). Dwindling gender gaps in employment and income have been named as likely drivers of declining marriage rates and increasing divorces as the proportion of women who can achieve upward mobility on the mating market declines (Wilson 1996, Stevenson and Wolfers 2007, Autor et al. 
2019). Indeed, couples are less likely to form, and are more likely to separate, when the woman's earnings approach the man's (Bertrand et al. 2013, Doumbia and Goussé 2019).

As with sex ratios and income inequality, it is reasonable to expect that the effects of gender inequality on mating competition will vary among individuals, depending on their status, age, and other characteristics. Here we model a simple scenario of hypergynous preference in order to extract predictions about the effects of varying gender gaps in wealth/status on the competition individuals experience and thus on their likely adaptive behaviour.

\section{Model}

In this paper we present a simple model to explore how the strength of within-sex competition for mates might be influenced by gender inequality, income inequality among men, and income inequality among women. We consider both competition among men and among women for mates in a heterosexual mating market. Our approach is to model the 'pairing success' of individual males and females in relation to their 'wealth' over a series of 'encounters'. Once an individual pairs (i.e. finds a mate), they do not go back into the pool for subsequent encounters; an individual either pairs monogamously or not at all.

We differentiate the related quantities of 'wealth' and 'status'. Status is the underlying percentile rank wealth of individuals within their sex, always ranging from 0 to 100 . Wealth is an absolute property that varies in relation to the distribution properties we assign, and it can thus differ between the sexes in both mean and variance.

The simplest form of our model applies a weak form of hypergyny in which women pair only with men of equal or greater wealth than themselves, and men mate with any woman willing to mate with them. We call this 'Choice Rule 1' or 'Strict Hypergyny'. We also modelled three other choice rules. Under Choice Rule 2 or 'Capped Hypergyny', women pair only with men who are wealthier than themselves, and men accept women whose wealth is no more than a specified number of units lower than theirs. This constitutes hypergyny within a stratified mating market, a pattern that has been documented in several societies (e.g., Dickemann 1979). Choice Rule 3 retains the same female preference for wealthier 
men and adds a second trait, $\mathrm{X}$, that men care about. Men accept any woman whose value of $X$ exceeds their own by a specified amount $\Delta X_{m}$ or more. Varying $\Delta X_{m}$ allows one to explore the effect of the strength of the male preference for $X$. Choice Rule 4 gives both women and men preferences for both wealth and X.

In the online supplementary material we explore some of the differences in model outcome that depend on the choice rules applied. In summary, when the rule components other than hypergyny are weak, the model results are very similar to those under Choice Rule 1. Stronger non-hypergyny components alter aspects of the model outcome but in most situations the important features of the model under Choice Rule 1 remain. Due to the qualitative similarity in results across the four types of choice rule, we present only the results of Choice Rule 1 in this manuscript to remain focussed on our main goal of exploring the consequences of hypergyny for pairing success and within-sex competition. We provide equivalent figures for each of the other three choice rules in the supplement.

Our interest in modelling this problem stems from the many consequences of within-sex competition. We equate an individual's low pairing success in our model with high competition to escape the circumstances that cause low paring success. Women who experience high competition are more likely than those who experience less competition to engage in competitive behaviours such as overt aggression, social exclusion, gossip (Fisher and Cox 2009), self-sexualisation and status-signalling (Blake et al. 2018, Blake and Brooks 2019) to improve their chances of attracting mates. Likewise, men strive to elevate themselves above other men, especially when they are in circumstances of poverty, youth, or low status that lower their mating success (Wilson and Daly 1997, Daly et al. 2001, Daly 2016, see also Greitemeyer and Sagioglou 2017).

Our model is an abstraction of the observation that women do tend to prefer men of higher wealth or status than themselves, whereas men compete to reach higher wealth and status in order to attract women. The preference/s underpinning human hypergyny are, obviously, far more complex and contingent than the simple preferences we model here. Nevertheless, simplification provides a useful first step towards understanding the behaviour of complex systems. 
All modelling and graphing presented is conducted in MATLAB. Our model considers four economic variables, the mean wealth for men $\left(\mu_{\mathrm{m}}\right)$, and women $\left(\mu_{\mathrm{f}}\right)$, and the standard deviation in wealth for men $\left(\sigma_{m}\right)$, and for women $\left(\sigma_{f}\right)$. Male and female wealth are each distributed normally, with given mean and standard deviation. We consider a large population ( 2 million individuals) with an even sex ratio, in which each individual is independently assigned a status (within-sex ranking) and this is converted into wealth by assigning a position in the relevant distribution.

Human mate choice occurs as an iterative process, with people who match progressively dropping out of the market, at least for a time. Individuals who experience low competition are more likely to find a mutually suitable match and thus to pair early on. Those who experience high competition will tend to match and mate later, or not at all. We therefore model human mating competition as a simulation with a given number of iterative rounds. Starting with an initial population where everyone is unpaired, men and women are randomly arranged into candidate pairs. If a pair is a 'match' (i.e. the candidates meet each other's choice criteria) both woman and man are henceforth recorded as paired. The remaining unpaired individuals are then repeatedly randomly arranged into new candidate pairs, for a specified number of iterations. Competition is then measured as the proportion of individuals that remain unpaired throughout the process.

The used Matlab code is provisionally available here, and will be made permanently available upon publication.

\section{Gender inequality}

When women and men have the same mean and standard deviation in wealth (i.e., $\mu_{m}=\mu_{f}$ and $\left.\sigma_{m}=\sigma_{f}\right)$, their initial pairing probability, in the first iteration of choice, follows a linear function of wealth rank (solid lines in Figure 1a), with the poorest men and the wealthiest women experiencing zero pairing success. 
Any difference from perfect equity (i.e., $\mu_{m} \neq \mu_{\mathrm{f}}$ ) results in the relationship between wealth rank and pairing success on the first iteration becoming non-linear. Dashed lines in Figure $1 \mathrm{a}$ show the relationship for a one SD difference between male and female mean wealth $\left(\mu_{\mathrm{m}}\right.$ $=55 ; \mu_{\mathrm{f}}=50$, and $\sigma_{\mathrm{m}}=\sigma_{\mathrm{f}}=5$ ). Such a gap alleviates competition (and thus elevates pairing success) for all women and men, but especially for the men in the lower half of the wealth rank distribution and women in the upper half. Whereas both women and men at 50 per cent wealth rank would be rejected by half the members of the opposite sex, the one S.D. difference in mean incomes lowers this value below 0.2 or twenty percent.

The number of iterations also influences competition within each sex. Figure $1 \mathrm{~b}$ shows the results of simulations for 10 iterations and Figure 1c for 100 iterations. More iterations result in fewer individuals of each sex remaining unpaired, since more pairing attempts results in more pairings. Iterative pairing - which is a realistic feature - results in almost all men of above average status and almost all women of below average status finding a match even when male and female mean wealth are equal. These reductions in competition are more dramatic when $\mu_{\mathrm{m}}>\mu_{\mathrm{f}}$.

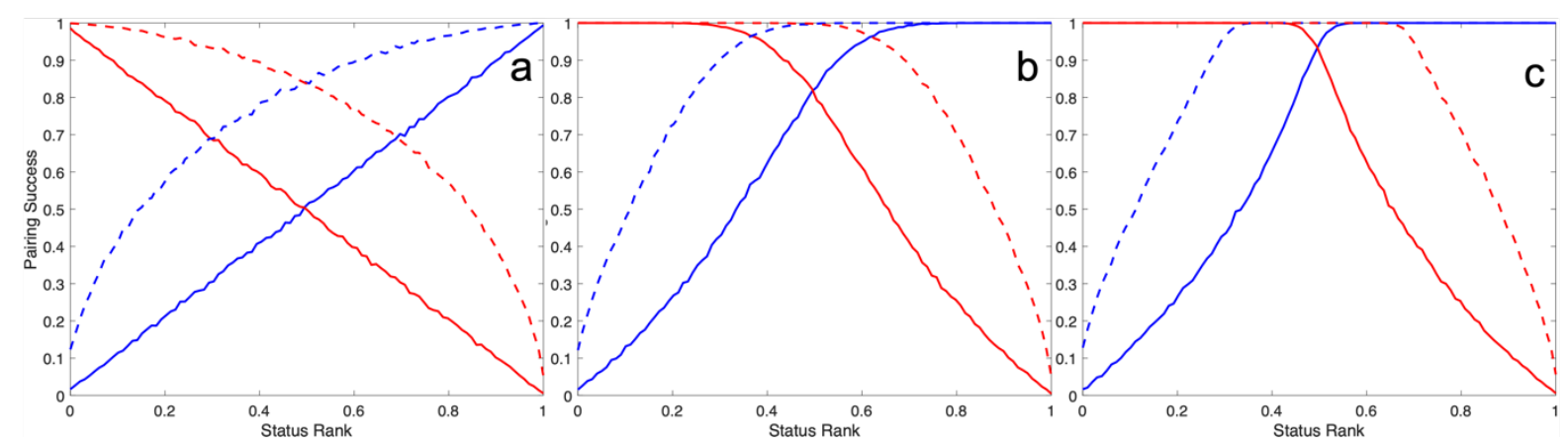

Figure 1. Pairing success of men (blue) and women (red) under equal mean wealth (solid) and a M-F gap of one standard deviation (dashed). Panels indicate the results from a. the first, $b$. the 10th and c. the 100th iteration. 
In order to explore how the size and direction of the gender gap effects the shape of these curves, we kept female mean wealth constant at $\mu_{\mathrm{f}}=50$ and allowed male mean income $\left(\mu_{m}\right)$ to vary by up to 4 S.D. in either direction (i.e., between 30 and 70). The more the average wealth gap favours men (i.e. higher values), the larger the proportion of both men and women who pair successfully under hypergyny (lighter shades in Fig 2a \& b). The opposite is true for female-biased wage gaps, where a growing proportion of men and women experience very low $(<0.1)$ pairing success (darker shades in Fig $2 a \& b$ ).

Figure 2 panels $\mathrm{c}$ and $\mathrm{d}$ show the extent to which pairing success increases (reds, positive numbers) or decreases (blues, negative numbers) compared with equity (i.e. Male-Female Wealth Gap $=0$ ). Both the lowest-status men and the highest-status women benefit most in terms of greater pairing success - from a male-biased gender gap in average earnings. High-status men and low-status women seldom remain unpaired under male-biased wealth gaps. The mating prospects of low-status men and high-status women remain largely the same - very poor - under female-biased wealth gaps. By contrast, female-biased average wealth gaps dramatically reduce the mating prospects of medium-high status men and medium-low status women who go from near-certainty to little hope of finding a match. 

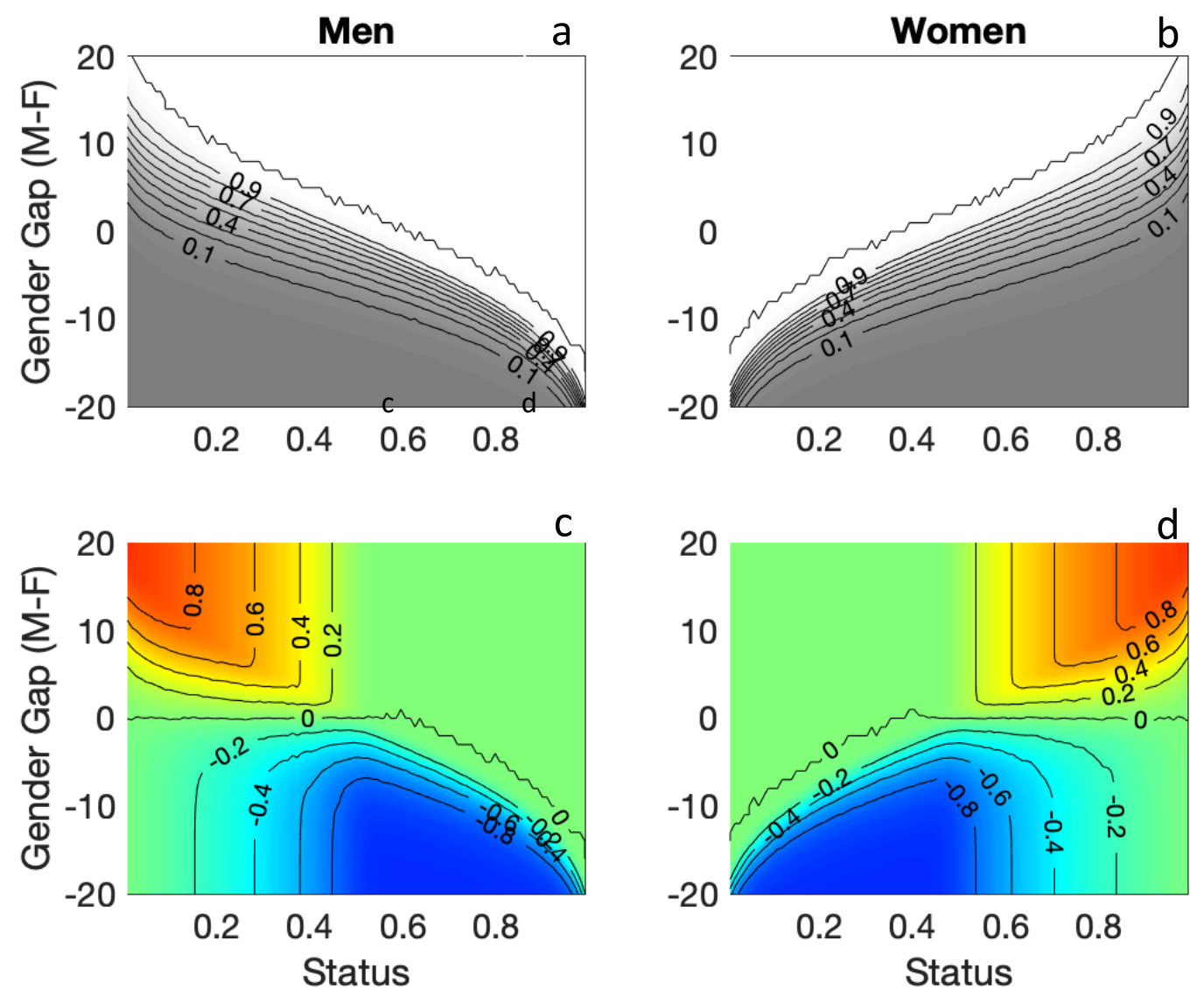

Figure 2. Pairing success experienced by men (a) and women (b), and difference from the situation of wealth equity for men (c) and women (d), in relation to status (i.e., wealth rank) and the size of the male-female wealth gap. In panels $a$ and $b$ the $z$-axis shows proportion of individuals paired after 100 encounters. In panels $c$ and d the z-axis shows pairing success at various levels of male-female gap minus pairing success at gender gap $=0$ (i.e. $\mu_{m}=\mu_{f}=50$ ).

\section{Income inequality}

Varying income inequality among men and among women at the same time (i.e., $\sigma_{m}=\sigma_{f}$ ) while there is no gender gap (i.e., $\mu_{m}=\mu_{\mathrm{f}}$ ) results in a straight line (as per solid lines in Figure 1a) at any modelled level of inequality. Once we introduce a gender gap (in the case of Fig. $\left.3, \mu_{m}=0.55, \mu_{f}=0.5\right)$, however, high inequality amplifies the relationship between pairing success and status, whereas low inequality diminishes that relationship. Another way of stating this result is that hypergyny generates a positive relationship between inequality and competition for mates.

Men of below-average wealth and women of above-average wealth experience stronger negative effects of high inequality (Fig $3 c$, d) relative to the baseline (i.e., $\sigma_{m}=\sigma_{f}=5$ ) 
inequality levels. These individuals do, however, experience some relief from competition under very low levels of inequality. Wealthy men's and poorer women's pairing success is very high (close to 100 percent, Fig 3 a \& b) and largely unperturbed by inequality in the form modelled in Figure 3.
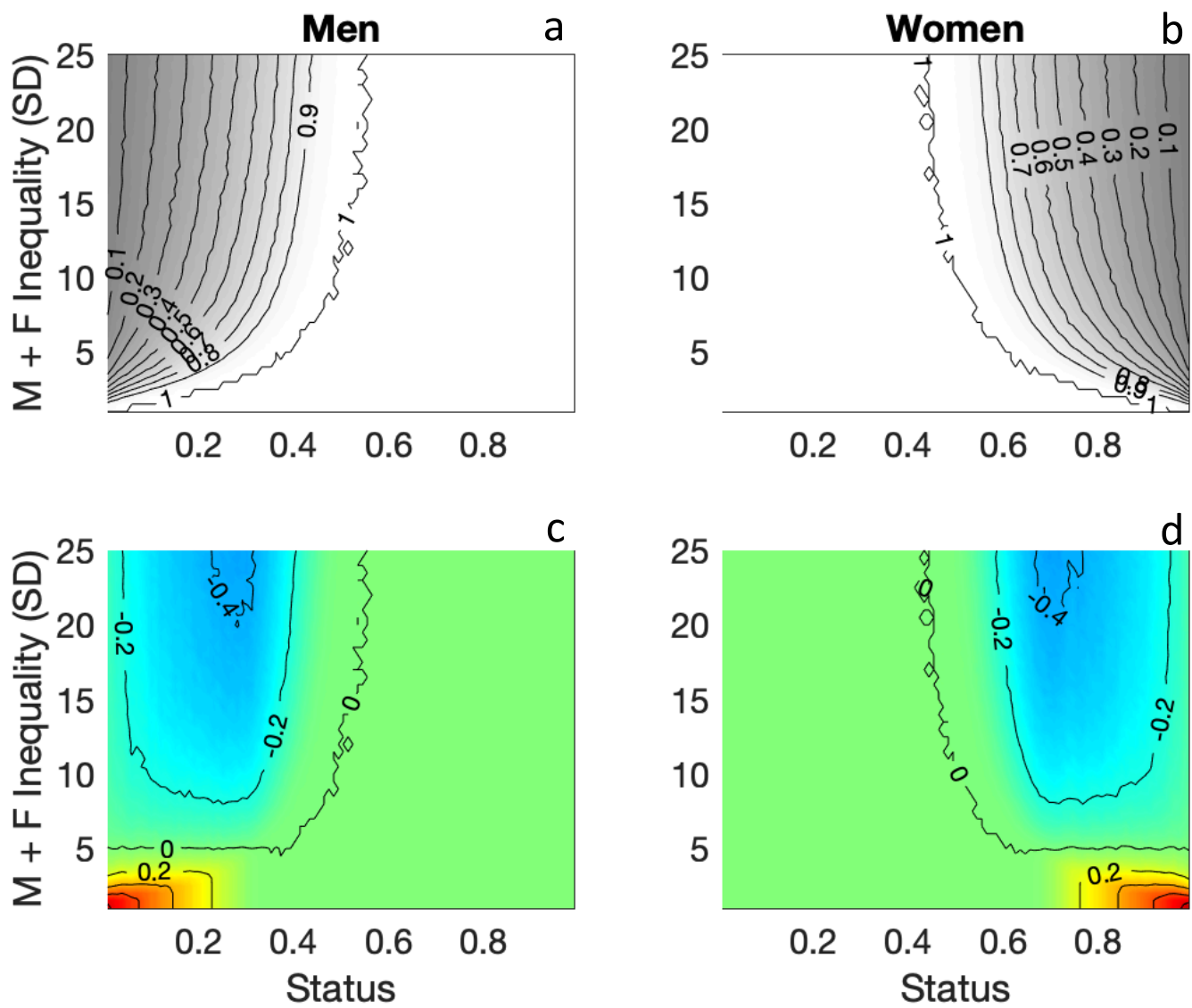

Figure 2. Pairing success (Z-axis) experienced by men and women in relation to status and inequality. Panels $a$ and $b$ show actual pairing success (after 100 encounters), and panels $c$ and $d$ show pairing success relative to that experienced under inequality of $\sigma_{m}=\sigma_{f}=5$. All figures includes a small male-female difference in mean $\mu_{m}=55, \mu_{f}=50$ for reasons explained in the text. 

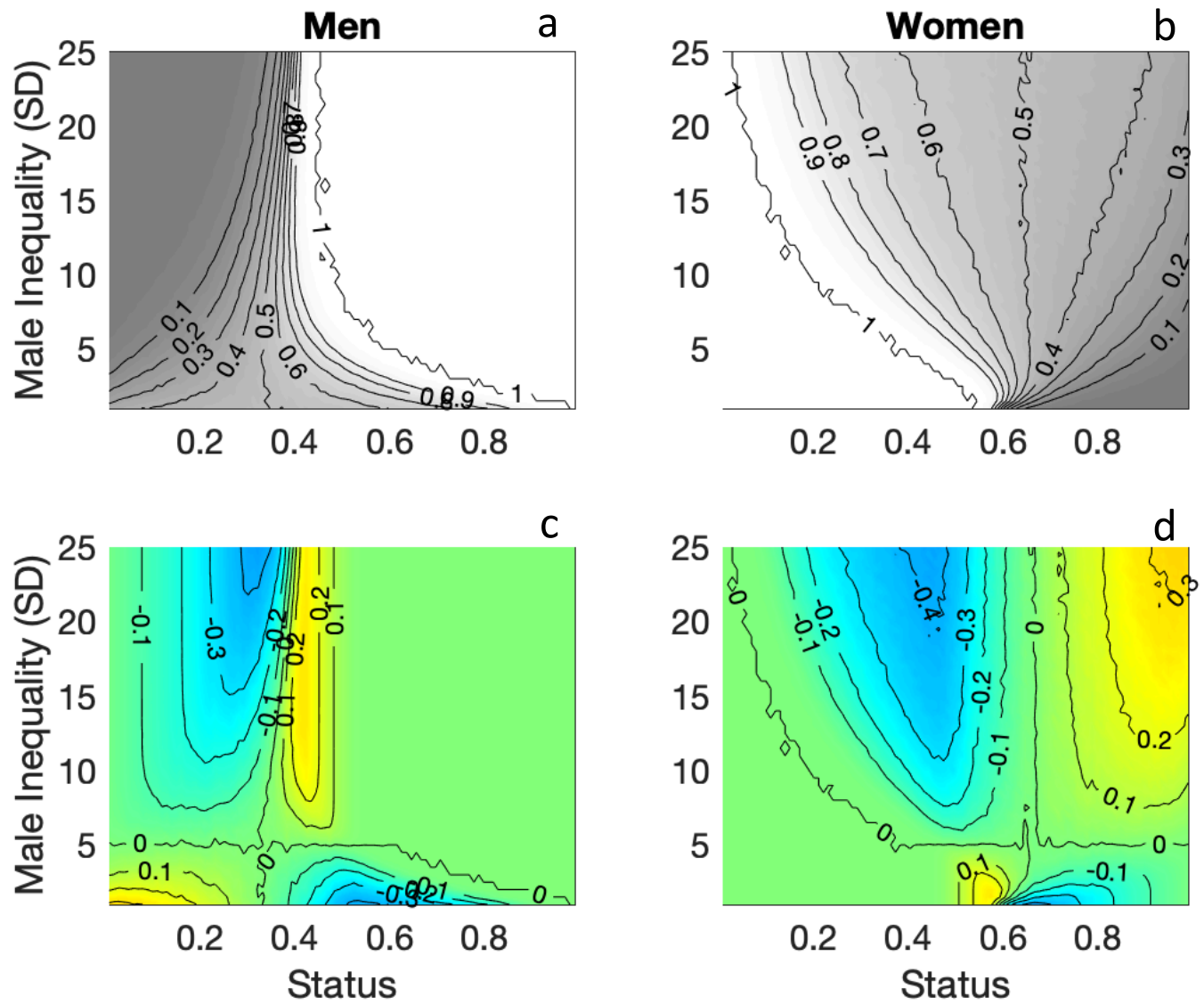

Figure 4. Pairing success (Z-axis) experienced by men and women in relation to status and wealth inequality among men. Panels $a$ and $b$ show pairing success (after 100 encounters), and panels $c$ and $d$ show pairing success relative to that experienced under $\sigma_{m}=5$ (i.e., equal male and female inequality). Male and female mean wealth is equal.

\section{Income inequality among men}

The effects of varying male inequality $\left(\sigma_{m}\right)$ on pairing success can be seen in Figure $4 a$ and $b$. The net effects of inequality compared with the null situation $\left(\sigma_{m}=\sigma_{f}=5\right)$ can be seen in Figure 4c (men) and d (women). Low inequality among men favours low-status men by increasing pairing success whereas high-status men experience lower pairing success. Under high inequality, medium-low status men are worse off, but medium-high status men are better off in terms of pairing success.

Among women, medium-low status women experience increased pairing success under low male inequality, and medium-high status women experience reduced pairing success. Under 
high male inequality, variation in pairing success is most strongly affected at the status extremes, with high-status women experiencing higher pairing success and low-status women experiencing lower pairing success than under intermediate male inequality $\left(\sigma_{m}=\sigma_{f}\right.$ $=5)$.

\section{Income inequality among women}

Varying female inequality $\left(\sigma_{f}\right)$ while keeping male inequality constant affected the pairing success of men and women (Figure 5) in ways that present a mirror image of the effects of varying male inequality. Low inequality among women improves pairing success of mediumhigh status men whereas medium-low status men experience lower pairing success. Under high inequality, low status men experience higher pairing success whereas high-status men experience lower pairing success than under intermediate male inequality $\left(\sigma_{m}=\sigma_{f}=5\right)$.

Among women, low status women experience lower pairing success and high-status women experience higher pairing success under low female inequality than they would under the intermediate baseline level. Under high among-female wealth inequality, medium-low status women experience higher and medium-high status women lower pairing success than they do under modest $\left(\sigma_{m}=\sigma_{f}=5\right)$ inequality. This effect arises because, as womens' wealth distribution broadens, its right tail overlaps less with the corresponding distribution for men. This makes it harder for medium-high women to find a match, leaving more partners for medium-low status women. 

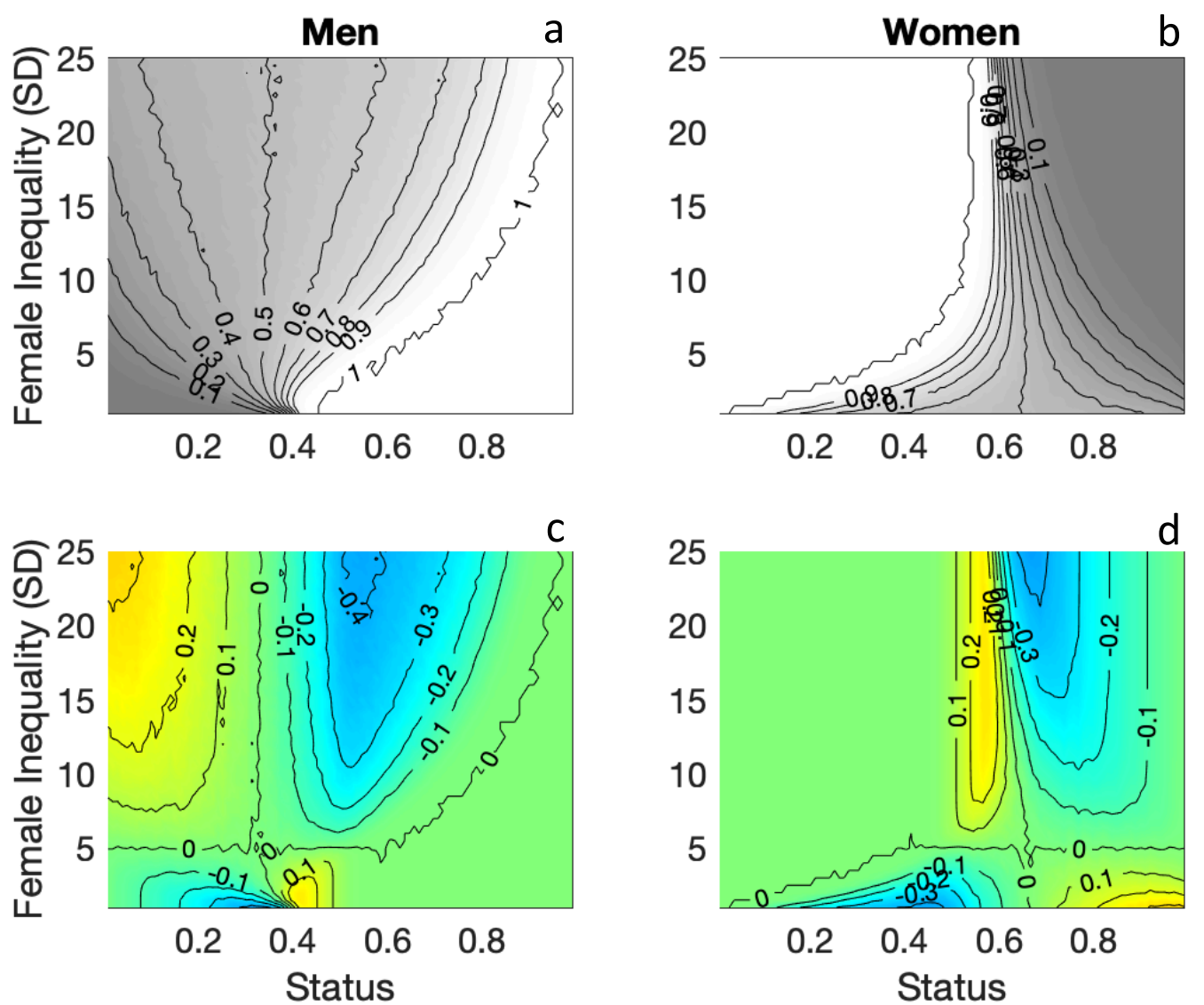

Figure 5. Pairing success (Z-axis) experienced by men and women in relation to status and wealth inequality among women. Panels $a$ and $b$ show pairing success (after 100 encounters), and panels $c$ and $d$ show pairing success relative to that experienced under $\sigma_{f}=5$ (i.e., equal male and female inequality). Male and female mean wealth is equal.

\section{Discussion}

The simple models that we present provide some adaptationist insights into the effects of a variety of economic inequalities on status-dependent pairing success of women and men. In general, under the kind of hypergyny modelled here, poorer men and wealthier women experience more competition for mates (i.e., lower paring success) than wealthy men and poorer women. When men earn more than women, on average, competition is alleviated and pairing success elevated in both sexes, and especially among poorer men and richer women. A dissection of the contributions of inequality within each sex reveals that high inequality within a sex or low inequality in the opposite sex tend to dampen the pairing success of members of high-competition groups (i.e., poorer men and wealthier women). 
Some of the predictions that our models generate correspond with observations from models that were designed for other purposes. Trivers and Willard (1973), for example, in modelling the evolution of parental investment in male versus female offspring, noted that a tendency for women to marry men of higher socioeconomic status than themselves can mean that the reproductive success of a man at the upper end of the socioeconomic scale is likely to exceed that of his sister, whereas the success of a woman of lower socioeconomic status is likely to exceed that of her brother. These differences in reproductive success arise due to status-dependent within-sex competition.

Likewise, Harpending and Rogers (1990), modelling the evolutionary consequences of wealth-transmission strategies within stratified societies, found that high status males have the highest long- term fitness, followed by low status females, then low status males and high status females. Adaptationist studies of dowry and bride-price practices find that dowry-indicative of high female-female competition for eligible mates-is more common at higher status strata, whereas bride price is more common at lower strata of the same societies (Dickemann 1979, Gaulin and Boster 1990).

Our models provide simple tools for understanding and interpreting empirical observations of mating market dynamics and their consequences. The greater competition and lower average mating success documented among men employed in the U.S. manufacturing sector during 1990-2014 as a result of the rise of Chinese manufacturing (Autor et al. 2013, Autor et al. 2017, Autor et al. 2019) provides a case in point. Shrinking male-female wage gaps and growing income inequality worsened the mating market prospects of men in areas impacted by this 'China Syndrome'. The relative importance of changes to male-female wage gaps and inequality remains unknown, but both processes are consistent with the predictions from our model (Autor et al. 2019). It is worth noting that men's worsening mating market prospects under the China syndrome had substantial effects on their broader communities, with men suffering poorer mental health, greater likelihood of addiction, suicide, and accidental death (Autor et al. 2019). Moreover, affected areas also showed greater political polarization (Autor et al. 2017). 
In a similar vein, we have found that high income inequality and low gender inequity, together with male-biased sex ratios or a dearth of single women, result in higher incidences of online misogyny of a kind that reflects local competition among men for mates (Blake et al. 2021, Brooks et al. in press). The number of tweets by or about men who consider themselves 'involuntary celibate' (i.e., 'Incel') is highest in parts of the USA where sex ratios are male-biased, there are few single women in the age range 18-39, where income inequality is highest, and where gender gaps in income are smallest (Brooks et al. in press). This work suggests that Incels are especially common, or at least active on social media, in places where large numbers of young men are unable to earn enough to attract any of the limited numbers of available women.

Our model suggests that poorer or lower-status men are more likely than wealthy or highstatus men to experience low mating success as a result of income inequality and narrowing gender gaps. Our model also suggests, however, that the two factors affect somewhat different groups of men; gender equity has the greatest effect on the poorest men, whereas inequality increases competition most for men who are just below average.

\section{Political considerations}

Gender gaps and economic inequality are well known to motivate ideological beliefs and political activity. In most cases, the political motivations surrounding inequalities are understood in terms of their financial consequences. People are motivated against or in favour of a particular form of inequality because of its effect on income or wealth per se. But if economic inequalities influence within-sex competition, then evolved psychological responses to mating market considerations may influence the political reactions to those inequalities.

Mating markets influence individual evolutionary fitness, and thus it should not surprise anybody that factors that influence mating markets also motivate individuals' political positions (Kurzban et al. 2010, Petersen et al. 2013, Hatemi et al. 2017, Petersen 2018, Hudson et al. 2020, Luberti et al. 2020). Individual differences in sexual strategies, notably openness to multiple, low-commitment sexual encounters are associated with less affinity 
for authoritarianism and for conservative political positions (Hatemi et al. 2017, Petersen 2018).

We predict that the changes in mating market competition that emerge from our model in response to varying inequality and gender inequity will motivate women's and men's political positions in ways that reflect their adaptive (i.e., evolutionary fitness) interests. The importance of status in a hypergynous system such as our simple model will throw the mating interests, and thus the political interests, of people of the same sex but different status into opposition. Such status-dependent individual differences are already known with regard to policies like income redistribution (Petersen et al. 2013, Luberti et al. 2020). Our models of status-dependent within-sex competition might be useful in discerning the interests of individual women and men in relation to changing patterns of income inequality or gender inequity.

Those interests might manifest as status-dependent attitudes concerning gender equity and economic inequality. They might also - or instead - manifest as status-dependent attitudes to hypergyny. We note that not only do Incels identify 'sexual inequality' as a proximate cause of their lack of mating success, but they also identify hypergyny as a more distal but pervasive cause of sexual inequality (see Brooks 2021, Perry and DeDeo 2021). Our models predict that opposition to hypergyny will be greatest among low-status men and very highstatus women. Their opposition to hypergyny will be more intense under gender equity than when men earn or own more than women. Men of above-average wealth and women of below-average wealth should be uniformly favourable to hypergyny under high male-female gaps but opposed to it when women out-earn men.

In a similar vein, opposition to hypergyny and/or to income inequality should be highest in moderately wealthy women or moderately poor men under high income inequality, when the degree of inequality among men and among women is the same. The effects are more variegated and complex when the degree of inequality is manipulated independently within each sex. In general, high inequality among men imposes greater mating-market competition on below-average men and women, whereas high inequality among women is more costly for above-average wealth individuals of both sexes. We hope that this 
modelling exercise will open up new opportunities within and beyond the evolutionary behavioral sciences for understanding the rich, contingent drivers of human behaviour, including political attitudes.

\section{Limitations}

The model we present here is intentionally simple. We added Choice Rules 2-4 (See Online Supplementary Material) to test whether our results were an artificial consequence of modelling only one simple female choice rule. Our model does not include an explicit time or search cost, but varying the number of iterations may have a similar effect. Neither does it consider feedbacks between economic circumstances, mating market performance, and the strength of preferences, particularly in relation to the risk of remaining forever unpaired. These, and the addition of a potential for serial monogamy or polygyny, would make for more complex models, and perhaps different results. We encourage researchers to extend the work we have done here to build a broader and more realistic picture of the phenomena in question.

\section{References}

Autor, D., D. Dorn, and G. Hanson. 2019. When work disappears: manufacturing decline and the falling marriage-market value of young men. American Economic Review: Insights 1:161-178.

Autor, D., D. Dorn, G. Hanson, and K. Majlesi. 2017. Importing political polarization: the electoral consequences of rising trade exposure. NBER WOrking Paper 22637.

Autor, D., D. Dorn, and G. H. Hanson. 2013. The China Syndrome: Local Labor Market Effects of Import Competition in the United States. American Economic Review 103.

Barber, N. 2000. On the relationship between country sex ratios and teen pregnancy rates: A replication. Cross-Cultural Research 34:26-37.

Becker, G. S. 1973. Theory of Marriage .1. Journal of Political Economy 81:813-846.

Becker, G. S. 1981. A treatise on the family. Harvard University Press, Cambridge, Mass.

Bertrand, M., J. Pan, and E. Kamenica. 2013. Gender identity and relative income within households. Quarterly Journal of Economics 130:571-614. 
Betzig, L. 1994. Sex, succession, and stratification in the first six civilizations. Pages 37-74 in L. Ellis, editor. Social Stratification and Socioeconomic Inequality. Praeger, Westport Conn.

Betzig, L. L. 1986. Despotism and differential reproduction : a Darwinian view of history. Aldine Pub., New York.

Blake, K. R., B. Bastian, T. F. Denson, P. Grosjean, and R. C. Brooks. 2018. Income inequality not gender inequality positively covaries with female sexualization on social media. Proc Natl Acad Sci U S A 115:8722-8727.

Blake, K. R., and R. Brooks. 2019. Income inequality and its implications for gendered conflict.in J. Jetten and K. Peters, editors. The Social Psychology of Income Inequality. Springer.

Blake, K. R., S. O'Dean, J. Lian, and T. F. Denson. 2021. Misogynistic tweets correlate with and prospectively predict domestic violence incidents over time. . Psychological Science in press, accepted October 2020.

Boone III, J. 1986. Parental investment and elite family structure in preindustrial states: A case study of late Medieval-early modern Portuguese colonies. . American Anthropologist 88:859-878.

Brooks, R. 2021. Artificial Intimacy: Virtual Friends, Digital Lovers and Algorithmic Matchmakers. NewSouth, Sydney.

Brooks, R. C., D. Russo-Batterham, and K. R. Blake. in press. Incel activity on social media linked to local mating ecology. Psychological Science.

Buss, D. M. 1991. Sex-Differences in Human Mate Preferences - Evolutionary Hypotheses Tested in 37 Cultures. Behavioral and Brain Sciences 14:519-519.

Buss, D. M. 1995. Psychological Sex-Differences - Origins through Sexual Selection. American Psychologist 50:164-168.

Buss, D. M., and D. P. Schmitt. 1993. Sexual Strategies Theory - an Evolutionary Perspective on Human Mating. Psychological Review 100:204-232.

Buss, D. M., T. K. Shackelford, L. A. Kirkpatrick, and R. J. Larsen. 2001. A half century of mate preferences: The cultural evolution of values. Journal of Marriage and Family 63:491503.

Chiappori, P. A., B. Fortin, and G. Lacroix. 2002. Marriage market, divorce legislation, and household labor supply. Journal of Political Economy 110:37-72. 
Chipman, A., and E. Morrison. 2013. The impact of sex ratio and economic status on local birth rates. Biology Letters 9 .

Cronk, L. 1991. Wealth, Status, and Reproductive Success among the Mukogodo of Kenya. American Anthropologist 93:345-360.

Daly, M. 2016. Killing The Competition: Economic Inequality and Homicide. Transaction Publishers, New Brunswick.

Daly, M., and M. Wilson. 2001. Risk-taking, intrasexual competition, and homicide. Nebraska Symposium on Motivation 47:1-36.

Daly, M., M. Wilson, and N. Vasdev. 2001. Income inequality and homicide rates in Canada and the United States. Canadian Journal of Criminology 43:219-236.

de Jong, K., E. Forsgren, H. Sandvik, and T. Amundsen. 2012. Measuring mating competition correctly: available evidence supports operational sex ratio theory. Behavioral Ecology 23:1170-1177.

Dickemann, M. 1979. The ecology of mating systems in hypergynous dowry societies. Social Science Information 18:163-195.

Doumbia, M., and M. Goussé. 2019. Gender Identity and Relative Income Within Households: Evidence from Canada. Centre de recherche sur les risques les enjeux économiques et les politiques ....

Edlund, L., H. Li, and J. Yi. 2010. Marriage and Crime: Evidence from China\&apos;s Rising Sex Ratios.

Emlen, S. T. 1976. Lek Organization and Mating Strategies in Bullfrog. Behavioral Ecology and Sociobiology 1:283-313.

Emlen, S. T., and L. W. Oring. 1977. Ecology, sexual selection, and the evolution of mating systems. Science 197:215-223.

Fisher, M., and A. Cox. 2009. The influence of female attractiveness on competitor derogation. J. Evol.Psych. 7:141-155.

Fromhage, L., and M. D. Jennions. 2016. Coevolution of parental investment and sexually selected traits drives sex-role divergence. Nature Communications 7:12517.

Gaulin, S. J., and J. S. Boster. 1990. Dowry as female competition. American Anthropologist 92:994-1005.

Goldin, C. D. 1990. Understanding the gender gap : an economic history of American women. Oxford University Press, New York. 
Greitemeyer, T., and C. Sagioglou. 2017. Increasing wealth inequality may increase interpersonal hostility: The relationship between personal relative deprivation and aggression. The Journal of Social Psychology 157:766-776.

Grossbard-Shechtman, A. 1984. A theory of allocation of time in markets for labour and marriage. The Economic Journal 94:863-882.

Guttentag, M., and P. F. Secord. 1983. Too many women? : the sex ratio question. Sage Publications, Beverly Hills.

Harpending, H., and A. Rogers. 1990. Fitness in stratified societies. Ethology and Sociobiology 11:497-509.

Hatemi, P. K., C. Crabtree, and R. McDermott. 2017. The relationship between sexual preferences and political orientations: Do positions in the bedroom affect positions in the ballot box? Personality and Individual Differences 105:318-325.

Hesketh, T., and Z. W. Xing. 2006. Abnormal sex ratios in human populations: Causes and consequences. Proceedings of the National Academy of Sciences 103:13271-13275.

Hopcroft, R. L. 2006. Sex, status, and reproductive success in the contemporary United States. Evolution and Human Behavior 27:104-120.

Hopcroft, R. L. 2021. High income men have high value as long-term mates in the U.S.: personal income and the probability of marriage, divorce, and childbearing in the U.S. Evolution and Human Behavior 42:409-417.

Hudson, V. 2002. A surplus of men, a deficit of peace: security and sex ratios in Asia's largest states. International Security 26:5-38.

Hudson, V. M., and A. M. d. Boer. 2004. Bare branches : the security implications of Asia's surplus male population. MIT Press, Cambridge, Mass.

Hudson, V. M., D. L. Bowen, and P. L. Nielsen. 2020. The first political order : how sex shapes governance and national security worldwide. Columbia University Press, New York.

Hughes, A. L. 1986. Reproductive success and occupational class in eighteenth-century lancashire, England. Social Biology 33:109-115.

Jetten, J., and K. Peters, editors. 2019. The Social Psychology of Income Inequality. Springer.

Kandrik, M., B. C. Jones, and L. M. DeBruine. 2015. Scarcity of female mates predicts regional variation in men's and women's sociosexual orientation across US states. Evolution and Human Behavior 36:206-210. 
Klug, H., J. Heuschele, M. D. Jennions, and H. Kokko. 2010. The mismeasurement of sexual selection. Journal of Evolutionary Biology 23:447-462.

Kokko, H., and M. D. Jennions. 2008. Parental investment, sexual selection and sex ratios. Journal of Evolutionary Biology 21:919-948.

Kokko, H., H. Klug, and M. D. Jennions. 2012. Unifying cornerstones of sexual selection: operational sex ratio, Bateman gradient and the scope for competitive investment. Ecology Letters 15:1340-1351.

Krahn, H., T. F. Hatnagel, and J. W. Gartrell. 1986. Income inequality and homicide rates: Cross-national data and criminological theories. Criminology 24:269-294.

Kurzban, R., A. Dukes, and J. Weeden. 2010. Sex, drugs and moral goals: reproductive strategies and views about recreational drugs. Proc Biol Sci 277:3501-3508.

Kvarnemo, C., and I. Ahnesjo. 1996. The dynamics of operational sex ratios and competition for mates. Trends in Ecology \& Evolution 11:404-408.

Lartey, S. T., R. Khanam, and S. Takahashi. 2016. The impact of household wealth on child survival in Ghana. Journal of Health, Population and Nutrition 35:38.

Luberti, F. R., K. R. Blake, and R. C. Brooks. 2020. Does the Quality of Mating Competitors Affect Socio-Political Attitudes? An Experimental Test. Adaptive Human Behavior and Physiology 6:501-531.

Lummaa, V. 2001. Reproductive investment in pre-industrial humans: the consequences of offspring number, gender and survival. Proceedings of the Royal Society of London.Series B. 268:1977-1983.

Marlowe, F. W., and J. C. Berbesque. 2012. The human operational sex ratio: Effects of marriage, concealed ovulation, and menopause on mate competition. Journal of Human Evolution 63:834-842.

Nettle, D., and T. V. Pollet. 2008. Natural Selection on Male Wealth in Humans. American Naturalist 172:658-666.

Peñaherrera-Aguirre, M., S. C. Hertler, A. J. Figueredo, H. B. F. Fernandes, T. Cabeza de Baca, and J. D. Matheson. 2018. A social biogeography of homicide: Multilevel and sequential canonical examinations of intragroup unlawful killings. Evolutionary Behavioral Sciences 2018:1-24.

Perry, C., and S. DeDeo. 2021. The cognitive science of extremist ideologies online. arXiv 2110.00626v1 [cs.SI]. 
Petersen, M. B. 2018. Reproductive interests and dimensions of political ideology. Evolution and Human Behavior 39:203-211.

Petersen, M. B., D. Sznycer, A. Sell, L. Cosmides, and J. Tooby. 2013. The Ancestral Logic of Politics: Upper-Body Strength Regulates Men's Assertion of Self-Interest Over Economic Redistribution. Psychological Science 24:1098-1103.

Price, M. E., N. Pound, and I. M. Scott. 2014. Female Economic Dependence and the Morality of Promiscuity. Archives of Sexual Behavior 43:1289-1301.

Reiss, I. L. 1986. A Sociological Journey into Sexuality. Journal of Marriage and the Family 48:233-242.

Schacht, R., and M. Borgerhoff Mulder. 2015. Sex ratio effects on reproductive strategies in humans. Royal Society Open Science 2.

Schacht, R., D. Tharp, and K. R. Smith. 2016. Marriage Markets and Male Mating Effort: Violence and Crime Are Elevated Where Men Are Rare. Human Nature 27.

Schmitt, D. P., and M. I. S. Descript. 2004. Patterns and universals of mate poaching across 53 nations: The effects of sex, culture, and personality on romantically attracting another person's partner. Journal of Personality and Social Psychology 86:560-584.

Shuster, S. M. 2016. Operational Sex Ratio. Pages 167-174 in R. M. Kliman, editor. Encyclopedia of Evolutionary Biology. Academic Press.

Shuster, S. M., and M. J. Wade. 2003. Mating Systems and Strategies. Princeton Universiy Press., Princeton, N.J. .

Stevenson, B., and J. Wolfers. 2007. Marriage and Divorce: Changes and their Driving Forces. Journal of Economic Perspectives 21:27-52.

Stone, E. A. 2018. Does Mate Scarcity Affect Marital Choice and Family Formation? The Evidence for New and Classic Formulations of Sex Ratio Theory. Marriage \& Family Review:1-20.

Trivers, R. L. 1972. Parental Investment and Sexual Selection. Pages 136-179 in B. Campbell, editor. Sexual Selection and the Descent of Man 1871-1971. Heinemann, London.

Trivers, R. L., and D. E. Willard. 1973. Natural selection of parental ability to vary the sex ratio of offspring. Science 179:90-92.

Van Leeuwen, M. H., and I. Maas. 2010. Historical studies of social mobility and stratification. Annual Review of Sociology 36:429-451. 
Voland, E. 1990. Differential Reproductive Success within the Krummhorn Population (Germany, 18th and 19th Centuries). Behavioral Ecology and Sociobiology 26:65-72. von Rueden, C. R., and A. V. Jaeggi. 2016. Men's status and reproductive success in 33 nonindustrial societies: Effects of subsistence, marriage system, and reproductive strategy. Proceedings of the National Academy of Sciences of the United States of America 113:10824-10829.

Weeden, J., M. J. Abrams, M. C. Green, and J. Sabini. 2006. Do high-status people really have fewer children? Education, income, and fertility in the contemporary US. Human Nature-an Interdisciplinary Biosocial Perspective 17:377-392.

Wilson, M., and M. Daly. 1985. Competitiveness, risk-taking, and violence - The young male syndrome. Ethology and Sociobiology 6:59-73.

Wilson, M., and M. Daly. 1997. Life expectancy, economic inequality, homicide, and reproductive timing in Chicago neighbourhoods. BMJ 314:1271.

Wilson, W. J. 1996. When Work Disappears: The World of the New Urban Poor. Alfred A. Knopf, New York.

Wooding, S., C. Ostler, B. R. Prasad, W. S. Watkins, S. Sung, M. Bamshad, and L. B. Jorde. 2004. Directional migration in the Hindu castes: inferences from mitochondrial, autosomal and Y-chromosomal data. Human Genetics 115:221-229.

Zhou, X. D., L. Li, Z. Yan, and T. Hesketh. 2013. High sex ratio as a correlate of depression in Chinese men. Journal of Affective Disorders 144:79-86. 